\title{
Setting up a myocardial perfusion scintigraphy service: clinical and business aspects
}

\author{
Prepared jointly by the British Cardiac Society, the British Nuclear Cardiology Society, and the \\ British Nuclear Medicine Society
}

\section{INTRODUCTION AND OVERVIEW}

Myocardial perfusion scintigraphy (MPS) has been available as a valuable tool in the investigation of patients with known or suspected coronary artery disease (CAD) for more than two decades. However, in notable contrast to the situation in the USA and parts of mainland Europe, MPS has been slow to establish itself in routine clinical practice in the UK. The National Institute for Health and Clinical Excellence (NICE) has recently completed a technology appraisal of MPS, with positive findings. The implication is that there ought to be an approximately fourfold increase in the provision of nuclear cardiology in England and Wales, with an estimated capital cost of $£ 18$ million and an annual revenue cost of $£ 27$ million.

MPS is an effective and cost effective investigation, but requires appropriate expertise and resources. The purpose of this document is to provide clinicians and managers who have little or no experience of MPS with the basic information required to begin planning a service. In all cases, advice should be sought from an experienced clinician and medical physics expert before any practical steps are taken.

In addition to MPS, several other non-invasive imaging modalities may be used to investigate patients with known or suspected CAD. In centres with expertise in other well established non-invasive techniques such as stress echocardiography (echo), it may be more appropriate to expand the existing service rather than attempt to set up a nuclear cardiology service "from scratch".

Nuclear medicine is probably the most regulated area of medicine, and several pieces of legislation apply. Involvement of a medical physics expert is mandatory. He/she will also provide scientific support for the non-clinical aspects of the service, including selection of acquisition protocols, data processing and analysis, and quality assurance (QA) for the gamma camera and other equipment.

MPS is indicated for the investigation of a large number of patients, and a service works most efficiently at high volume. Reliance on outside space, staff, or equipment can affect the smooth flow of patients, and should be avoided where possible. When planning an MPS service, adequate space should be identified, and resources allocated to provide all necessary equipment and appropriate levels of staffing. Purchase of a gamma camera is likely to be the largest capital outlay, and a formal tender process is necessary. The camera's specifications will be determined largely by whether it will be used exclusively for MPS, or whether it will also perform general nuclear medicine procedures.

A number of types of expertise must be brought together to perform MPS, and the national shortage of trained medical, technical, and physics staff is one of the greatest challenges to expansion. There are insufficient numbers of nuclear physicians and radionuclide radiologists, and very few cardiologists have adequate advanced training in nuclear cardiology, including those with a subspecialist interest in non-invasive imaging. There is also a lack of appropriately trained non-medical staff to cover not only the traditional functions of radiopharmaceutical administration and imaging, but also the increasing demand for stress testing led by technical or nursing staff.

Procedures for MPS are varied, reflecting the fact that all methods have advantages and disadvantages. Each department should develop a protocol that best suits its local requirements, and nationally agreed procedure guidelines are available.

It may be impossible for many hospitals keen to establish an MPS service to assemble the necessary facilities and expertise in the short term. In such cases a "hub and spoke" approach may prove useful. Initially, resources would be directed at an existing large nuclear medicine department in a neighbouring hospital which would provide the service. Subsequently, it might become increasingly cost effective for a referring hospital to set up its own service locally, with support provided from the larger department. An alternative model would be the use of staffed mobile nuclear cardiology laboratories as a bridge to a hospital setting up its own service, analogous to the use of mobile cardiac catheterisation laboratories.

Setting up MPS requires a close partnership between a number of hospital departments and professional groups. There may be scepticism and rivalry to be overcome, and a planned stepwise approach to establishing and consolidating the nuclear cardiology service may avoid early failure.

Abbreviations: AC, attenuation correction; ALARA, as low as reasonably achievable; ARSAC, Administration of Radioactive Substances Advisory Committee; ASNC, American Society of Nuclear Cardiology; BCS, British Cardiac Society; BNCS, British Nuclear Cardiology Society; BNMS, British Nuclear Medicine Society; CAD, coronary artery disease; CCST, certificate of completion of specialist training; CHAl, Committee for Healthcare Audit and Improvement; CMR, cardiac magnetic resonance (imaging); CT, computed tomography; DEFRA, Department of Environment, Food and Rural Affairs; DGH, district general hospital; EA, Environment Agency; EBCT, electron beam computed tomography; echo, echocardiography; EMPIRE, economics of myocardial perfusion imaging in Europe; HSE, Health and Safety Executive; IRMER, lonising Radiation (Medical Exposures) Regulations; IRR, lonising Radiations Regulations; IPEM, Institute of Physics and Engineering in Medicine; JCHMT, Joint Committee for Higher Medical Training; MARS, Medicines (Administration of Radioactive Substances) Regulations; MCE, myocardial contrast echocardiography; MPS, myocardial perfusion scintigraphy; nGMS, new General Medical Services (Contract); NHS, National Health Service; NICE, National Institute for Health and Clinical Excellence; NatPACT, national primary and care trust; NPC, national prescribing centre; NSF, National Service Framework; PACS, picture archiving communication systems; PALS, Patient Advice and Liaison Service; PCT, primary care trust; PEC, Professional Executive Committee; PET, positron emission tomography; QA, quality assurance; RAMRoad, Radioactive Materials (Road Transport) Regulations; RIS, radiology information systems; RPA, radiation protection advisor; RPS, radiation protection supervisor; RSA, Radioactive Substances Act; SON, summary of need; SPECT, single photon emission computed tomography; ${ }^{99 \mathrm{~m}} \mathrm{Tc}$, technetium- $99 \mathrm{~m} ;{ }^{201} \mathrm{Tl}$, thallium-201; WTE, whole time equivalent 
Once a plan has been developed for the establishment of a nuclear cardiology service, funding will be required. This will almost certainly have to come from primary care trusts (PCTs), probably via a local NICE Implementation Committee. PCTs do not receive "ring fenced" money to cover NICE recommendations, and the positive appraisal of MPS will not automatically trigger funding. A carefully constructed business plan will be necessary, and a template is provided in electronic format to complement this document.

\section{NICE TECHNOLOGY APPRAISAL 73}

NICE published its technology appraisal guidance number 73 in November 2003, entitled "Myocardial perfusion scintigraphy for the diagnosis and management of angina and myocardial infarction". ${ }^{1}$ MPS was found to be both clinically effective and cost effective in this role. Although not included in the scope of the appraisal, it is also effective in the assessment of heart failure, particularly for the detection of myocardial viability and hibernation. ${ }^{2}$

NICE recommended that MPS should be used:

- as the initial diagnostic tool for people with suspected CAD for whom the exercise ECG poses particular problems, including those with conduction abnormalities on the resting ECG, those unable to exercise, women, and diabetics

- as part of an investigational strategy for the diagnosis of suspected CAD in people with lower likelihood of CAD

- as part of an investigational strategy in symptomatic patients after myocardial infarction or revascularisation.

NICE referred to professional evidence that approximately 1200 MPS studies per million population per year are currently performed in the UK, but that 4000 would be a more appropriate level of provision based on targets for revascularisation procedures and invasive coronary angiography. Suitable waiting times would be six weeks for routine studies and one week for urgent studies, rather than the current national average of 20 weeks.

To achieve these levels of provision and accessibility, it is estimated that 73 gamma cameras would be needed in England and Wales at an estimated capital cost of $£ 18$ million and an annual revenue cost of $£ 27$ million. This expansion will need to be phased over several years because of the shortage of trained personnel.

Since publication of the NICE guidance, the profession has taken several steps to assist its implementation, including publication of the evidence for MPS and procedure guidelines, and this has led to considerable interest from providers and PCTs in establishing or expanding an MPS service. ${ }^{3-5}$

\section{WHY CHOOSE MPS OVER OTHER IMAGING MODALITIES?}

\subsection{Introduction}

The National Service Framework (NSF) document on CAD has focused attention on the efficient risk assessment of patients presenting with chest pain. Many rapid access chest pain clinics have been set up, typically offering a clinical assessment and exercise ECG, followed by an immediate decision as to whether to offer invasive coronary angiography. In practice, a non-invasive imaging investigation such as MPS is seldom requested, even in situations where the exercise ECG is known to be inaccurate (abnormal resting ECG, inability to exercise, women), despite the guidelines of the British Cardiac Society (BCS). ${ }^{6}$ Such patients have a high probability of normal coronary angiography-for example, $56 \%$ in women assessed in a chest pain clinic in Southampton compared with only $16 \%$ in men. ${ }^{7}$
A non-invasive imaging investigation may be of value in a number of clinical situations:

- diagnosis of CAD

- prognostication in known/suspected CAD

- assessment of acute chest pain

- identification of culprit lesion following angiography

- investigation of heart failure, including evaluation of hibernating myocardium.

\subsection{Alternatives to MPS}

Many patients would benefit from some form of non-invasive imaging before (or even after) coronary angiography. MPS is one such investigation, but there are several alternatives:

- Echo

- stress echo

- myocardial contrast echo (MCE)

- Cardiac magnetic resonance (CMR) imaging

- stress CMR

- perfusion CMR

- Positron emission tomography (PET)

- Computed tomographic (CT) imaging

- CT coronary calcium imaging (including electron beam CT: EBCT)

- multislice CT coronary angiography

\subsection{Advantages of MPS}

In a given centre, with specific expertise and research interests, there may be good reasons to favour another imaging investigation over MPS. However, all else being equal, MPS has a number of important advantages over its alternatives $^{3}{ }^{4}$ :

- Large body of supporting clinical literature - This extends back over more than two decades, and supports the use of MPS in all its variations.

- High diagnostic accuracy for CAD-Sensitivity 80-90\%, depending on the population studied. Normalcy rate $90 \%$.

- Excellent prognostic power-There is a remarkable consistency across the literature. A normal MPS study predicts a risk of cardiac death or non-fatal myocardial infarction of $0.6 \%$ per year for at least five years. An abnormal study predicts a risk which increases in proportion to the degree of abnormality.

- Ability to deliver a high volume service-A single dedicated cardiac gamma camera can be used to investigate approximately 2000 patients per year, with relatively little requirement for hands-on consultant input (a scarce resource in the UK). It should nevertheless be acknowledged that there are also some very high volume stress echo centres in the USA.

- Applicable to all patients - Imaging is possible in all but the most morbidly obese patients (because of weight limitations on the imaging table), for whom cardiac catheterisation would be similarly impossible. There are no difficulties with imaging windows (cf echo). Small cardiac gamma cameras with open gantries are suitable for claustrophobic patients, and pose no problems for those with pacemakers and other metal implants ( $\mathrm{cf} C \mathrm{CMR}$ ).

- Operator independent-There is good agreement between observers in reporting. ${ }^{8}$ 
- Published evidence of cost effectiveness-Diagnostic strategies that involve MPS, whether as a first line investigation or following an equivocal exercise ECG, are cheaper than those that do not. ${ }^{10}$ In the EMPIRE (economics of myocardial perfusion imaging in Europe) study, a diagnostic strategy which utilised MPS reduced the management cost per patient over a two year period by $14 \%$ for those with CAD and by 33\% for those without CAD. There was no difference in clinical outcome.

\section{SETTING UP AN MPS SERVICE}

\subsection{Radiation protection, regulatory issues, and the role of medical physics}

4.1.1 Regulatory issues

Nuclear medicine is probably the most regulated area of medicine. It involves the use of radiation and therefore the Ionising Radiation Regulations 1999 and the Ionising Radiations (Outside Workers) Regulations 1993 apply. The majority of this radiation comes from radioactive material rather than X-ray devices. Hence the Radioactive Substances Act 1993 and the Radioactive Material (Road Transport) Regulations 1996 come into play. Furthermore this radioactive material is being used for the diagnosis and treatment of patients and is therefore covered by the Ionising Radiation (Medical Exposures) Regulations 2000 and also the Medicines (Administration of Radioactive Substances) Regulations 1978/1995. Finally in these days of global terrorism, the Police Counter Terrorism units are taking an interest in the use and storage of radioactive material in hospitals.

\section{Medicines (Administration of Radioactive Substances) Regulations 1978/1995 (MARS)}

This is administered by the Department of Health through the Administration of Radioactive Substances Advisory Committee (ARSAC). It is a requirement that the administration of radioactive substances for diagnosis, therapy or research must be the responsibility of a medical practitioner who holds a certificate issued by the Department of Health. This is commonly called an ARSAC certificate. All work must be covered by a certificate. Before a certificate is issued, the ARSAC committee will consider the training and experience of the applicant in the clinical use of radioactive substances and in radiation protection, and also the infrastructure and support in place (equipment, radiation protection adviser, medical physics expert)

\section{lonising Radiations Regulations 1999 (IRR99)}

These are administered by the Health and Safety Executive (HSE) and are primarily concerned with the protection of staff but also cover source security and record keeping. Some of the many requirements are:
- inform HSE of the use of radiation
- prior risk assessment
- local rules for radiation protection
- radiation protection advisor (RPA)
- radiation protection supervisor (RPS)
- source security
- controlled areas
- contingency plans.

lonising Radiation (Medical Exposures) Regulations 2000 (IRMER)

These are administered by the Department of Health and are intended to ensure the protection of the patient or research subject and any comforters and carers who may also be exposed to radiation. The regulations put specific responsibilities on the employer such as the requirements for defined procedures and protocols. They also define various roles and the responsibilities of those roles. These are the referrer, practitioner, operator and medical physics expert.

\section{Radioactive Substances Act 1993 (RSA93)}

This Act was developed by the Department of Environment, Food and Rural Affairs (DEFRA) and is regulated by the Environment Agency (EA). Its purpose is primarily the protection of the environment from radioactive discharge. The EA regulates the holding and disposal of radioactive material. It is necessary to obtain registrations for both closed sources (for QA, etc) and open sources (radiopharmaceuticals, etc), and an authorisation to accumulate and dispose of radioactive waste. It is relatively straightforward to obtain the licences to hold activity, but an application for an authorisation for disposal is both complex and expensive. In addition to stating how much waste will be produced, it is necessary to perform an assessment of the impact of the discharge and to provide a "best practicable means" document that demonstrates the measures being applied to minimise the production of waste. EA inspectors will expect to see a management system in place and will review source security and working, storage and disposal conditions.

\section{Radioactive Materials (Road Transport) Regulations 1996 (RAMRoad)}

This legislation is only relevant if radioactive material is being transported, and it is the duty of the sender to ensure it is applied. The legislation calls for a quality system, driver training, and a safety adviser. The regulations are not applicable to those sites which receive their radiopharmaceuticals from elsewhere and do not themselves transport anything.

Anyone providing a nuclear cardiology service must ensure that they are operating within the framework of all of the above regulations. It is unlikely that anyone setting up a new service would be starting completely "from scratch". There are few hospitals that do not have an $x$ ray department, and therefore some of the regulatory infrastructure is already likely to be in place. If there is a nuclear medicine department, then almost everything else will be covered. Involvement of medical physics at an early stage is absolutely essential: there are legal requirements to have both an RPA and a medical physics expert (these terms are defined in the appropriate regulations) and these individuals will be experienced in working with and applying the appropriate regulations.

\subsubsection{Radiation protection: practical issues}

In contrast to some other non-invasive imaging techniques, notably stress echo and CMR, MPS involves exposure to ionising radiation. Table 1 sets out the approximate radiation exposure and consequent increase in lifetime risk of fatal cancer for the various MPS protocols. For comparison, the exposure of patients undergoing MPS with a technetium$99 \mathrm{~m}$-based tracer is typically the equivalent of 250 plain chest $x$ rays.

A full coverage of radiation protection issues is beyond the scope of this document. The legislation sets the framework in which nuclear cardiology must function, but the practical aspects of radiation protection can be reduced to the concept of ALARA: keeping exposure "as low as reasonably achievable". The methods by which this is achieved rely upon the knowledge and training of the individuals involved. It is fundamental to much of the legislation that those involved with radiation are "competent", that their competence is assessed, and that they are involved in continuous 
Table 1 Radiation exposure and risk associated with different MPS protocols

\begin{tabular}{|c|c|c|c|c|}
\hline Radiopharmaceutical & Protocol & $\begin{array}{l}\text { Dose } \\
\text { (MBq) }\end{array}$ & $\begin{array}{l}\text { Effective dose } \\
\text { equivalent (mSv) }\end{array}$ & $\begin{array}{l}\text { Lifetime risk of } \\
\text { fatal cancer }\end{array}$ \\
\hline \multirow[t]{2}{*}{ Thallium-201 } & Stress redistribution & 80 & 18 & 1 in 1389 \\
\hline & Stress reinjection & 120 & 27 & 1 in 926 \\
\hline \multirow[t]{2}{*}{${ }^{99 m}$ Tc-sestamibi } & Two day & 800 & 10 & 1 in 2500 \\
\hline & One day & 1000 & 13 & 1 in 2083 \\
\hline \multirow[t]{2}{*}{${ }^{99 m}$ Tc-tetrofosmin } & Two day & 800 & 8 & 1 in 3125 \\
\hline & One day & 1000 & 10 & 1 in 2500 \\
\hline
\end{tabular}

professional development and remain competent. The RPA can usually help to provide training.

\subsubsection{QA of gamma cameras and other equipment} A system of QA must be in place. This involves not only the gamma camera, but also the dose calibrator used to measure the radiopharmaceuticals, and contamination and dose rate monitors. Gamma cameras must be rigorously tested on installation before acceptance. Like other complex imaging equipment they are subject to both sudden failure and gradual degradation in image quality: routine daily checks should be performed by a technologist or radiographer, with periodical detailed tests by a medical physicist.

\subsubsection{Some questions to ask when planning an MPS service}

- Do I have appropriate training and experience? If not who does, or how do I get it?

- Is there already an $x$ ray department in the hospital (a head start), or even better a nuclear medicine department? How can I work with them or use them? Will nuclear cardiology be offered as part of an integrated nuclear medicine service or as a stand alone service?

- Is there a Medical Physics Department in the hospital? Is there an appropriately qualified RPA/"medical physics expert"? If not, from where will support come?

- Is there an on-site radiopharmacy? If not, from where will the radiopharmaceuticals come?

\subsection{Space requirements}

The location of the nuclear cardiology service is important, but is more likely to be determined by availability of space in a given hospital than by practical considerations. Unless the service is to be provided out of an existing nuclear medicine department, thought should be given to the proximity of other departments which may be needed to supply staff and facilities (cardiology, nuclear medicine), and radiation protection issues (for example, placing the department next to the antenatal clinic may be undesirable!).

The requirements for space will vary from hospital to hospital, and will depend particularly on whether nuclear cardiology is to be provided as part of an integrated general nuclear medicine service, or whether a "stand alone" service is required. MPS is potentially valuable for a large number of patients, and is best delivered as an efficient, high volume service. Wherever possible, a nuclear cardiology service should be self contained with its own dedicated stress, radiopharmaceutical handling, and imaging facilities. This limits the effect of external factors on the smooth running of the department, upon which high patient throughput depends. Some space requirements are suggested in table 2. These are idealised and could be reduced where space is at a premium.

\subsection{Equipment requirements \\ 4.3.1 Overview}

Specialised equipment will not be the only capital expenditure required to establish a nuclear cardiology department. Advice will be required from a medical physics expert and the gamma camera manufacturer regarding any necessary refurbishment (for example, floor levelling, floor coverings, lead shielding). In addition, air conditioning will be essential in the camera room and highly desirable in the stress room. Furnishings will be required as for any hospital department. Table 3 lists essential pieces of equipment, together with some estimated prices.

\subsubsection{Choosing a gamma camera}

The first decision to be made when buying a new gamma camera is whether it will be used exclusively or only part time for MPS, and in the latter case which non-cardiac studies are planned for the camera. A small field-of-view multi-detector camera with a fixed detector configuration (typically two heads at $90^{\circ}$ ) is ideal as a dedicated cardiac camera, and costs approximately $£ 120-160 \mathrm{k}$. Where more flexibility is required, a large field-of-view multi-detector camera with detectors that can be used in variable configurations is a better choice, and costs up to $£ 250 \mathrm{k}$. It should be noted that while a small cardiac camera provides less flexibility for non-cardiac work than a full sized camera, image quality for MPS studies is indistinguishable.

Attenuation correction (AC) for MPS is now widely available, but at significant additional cost. Where external

Table 2 Idealised space requirements for a nuclear cardiology service

\begin{tabular}{|c|c|c|}
\hline Room & Comment & $\begin{array}{l}\text { Space } \\
\left(\mathrm{m}^{2}\right)\end{array}$ \\
\hline Camera room & $\begin{array}{l}\text { Dedicated cardiac camera can now fit } \\
\text { into space as small as } 6.5 \times 9.5 \text { feet! }\end{array}$ & 12 \\
\hline Stress room & $\begin{array}{l}\text { Depends on how much equipment is } \\
\text { required }\end{array}$ & 12 \\
\hline $\begin{array}{l}\text { Injection/recovery } \\
\text { room }\end{array}$ & $\begin{array}{l}\text { Useful to prepare patients for stress, } \\
\text { inject at rest, or monitor following } \\
\text { adverse event }\end{array}$ & 6 \\
\hline Hot lab & Storage and handling of doses and waste & 6 \\
\hline Hot lavatory & For regulatory reasons & 2 \\
\hline $\begin{array}{l}\text { Patient waiting } \\
\text { area }\end{array}$ & $\begin{array}{l}\text { Must have specific area for injected } \\
\text { patients }\end{array}$ & 10 \\
\hline $\begin{array}{l}\text { Reception/front } \\
\text { office }\end{array}$ & $\begin{array}{l}\text { Handle appointments and patients, } \\
\text { issue reports }\end{array}$ & 8 \\
\hline $\begin{array}{l}\text { Reporting room/ } \\
\text { back office for staff }\end{array}$ & $\begin{array}{l}\text { Could be combined with front office } \\
\text { if space limited }\end{array}$ & 10 \\
\hline Total (ideal) & & 66 \\
\hline Total (limited) & & 52 \\
\hline
\end{tabular}

Figures are based on a stand alone department, but there would be important savings if the service were to be provided out of an existing nuclear medicine department.

Space that could be saved if space is highly restricted is italicised. 
Table 3 Essential equipment to establish a nuclear cardiology service

\begin{tabular}{|c|c|c|}
\hline Equipment & Comment & Price \\
\hline $\begin{array}{l}\text { Dedicated cardiac } \\
\text { gamma camera }\end{array}$ & $\begin{array}{l}\text { Small field-of-view, heads fixed } \\
\text { at } 90^{\circ} \mathrm{NB} \text { service contract } \\
\text { approx 8-10\% of purchase } \\
\text { price, i.e. £10-16k per year }\end{array}$ & $£ 120-160 k$ \\
\hline $\begin{array}{l}\text { Treadmill/ergometer } \\
\text { and ECG system }\end{array}$ & & $£ 20 \mathrm{k}$ \\
\hline $\begin{array}{l}\text { Defibrillation and } \\
\text { resuscitation trolley }\end{array}$ & & $£ 9 k$ \\
\hline $\begin{array}{l}\text { Rapid infusion } \\
\text { syringe pump }\end{array}$ & $\begin{array}{l}\text { For adenosine or dobutamine } \\
\text { stress }\end{array}$ & $£ 2 k$ \\
\hline Dose calibrator & $\begin{array}{l}\text { To draw up or check doses of } \\
\text { radiopharmaceutical }\end{array}$ & $£ 4 k$ \\
\hline Assorted lead shields & $\begin{array}{l}\text { Lead shield for handling doses, } \\
\text { lead coffins, syringe shields, } \\
\text { shielded pedal bin and } \\
\text { sharps bin }\end{array}$ & $£ 2 k$ \\
\hline Radiation detector & $\begin{array}{l}\text { For routine monitoring or in } \\
\text { the event of a spillage }\end{array}$ & $£ 1 k$ \\
\hline Total & & $\leqslant £ 200 k$ \\
\hline
\end{tabular}

Figures are based on a stand alone department, but there would be important savings if the service were to be provided out of an existing nuclear medicine department.

The prices shown are estimates given for planning purposes only. In all cases, manufacturers should be approached to provide accurate quotations.

radionuclide transmission sources are used, the cost of replacing sources must be borne in mind. $X$ ray based systems are a one-off purchase, but are generally more expensive, larger, and heavier. The use of AC imposes additional burdens on a department in terms of camera QA and the complexity of reporting studies. Staff must be confident that the expertise exists within the department to meet these challenges when deciding whether to buy a camera with AC.

An acquisition computer will be required alongside the gamma camera, and should be purchased from the same manufacturer. It is probably also best to buy the processing workstation from the same manufacturer, though the trend for these to become PC based may allow centres to look elsewhere for equipment that precisely suits their needs in future. Connectivity to existing computer workstations, radiology information systems (RIS), and picture archiving communication systems (PACS) varies between manufacturers and requires careful consideration. Data can be archived to one of a number of different storage media, including computer discs, magneto-optical discs, and DVDs. Large volume data storage can be effectively achieved using an archive server, but this is seldom available.

\subsubsection{Tender process for buying a gamma camera}

The tender process is basically the same whether a centre intends to purchase or lease a gamma camera. Once funding is secured, a specification should be prepared using the BNMS gamma camera tender questionnaire (www.bnms. org.uk). The first step is to produce a summary of need (SON), an example of which is contained within part A of the tender questionnaire. Next an advertisement is placed in the European Journal using the SON. Once expressions of interest have been received, pre-tender meetings should be held with selected suppliers, and demonstration visits arranged involving senior physics, technology, and medical staff. Pre-tender meetings help to make the formal part of the tender process (the exchange and evaluation of tender documents between purchaser and supplier) as straightforward and short as possible (typically 4-5 weeks).

Demonstration visits should concentrate on practical issues that are difficult to convey in a written document:
- patient comfort during image acquisition

- user friendliness of equipment

- speed of equipment set up

- ease and speed of collimator exchange

- first hand, unbiased information about camera reliability

- speed of service response following equipment failure

- critical appraisal of proprietary software for processing and display

- critical appraisal of patient database management system.

Tender documents are then issued using parts B, C and E of the tender questionnaire, along with National Health Service (NHS) supplies maintenance documents which are mandatory locally. Suppliers respond by completing the questionnaire, indicating whether specific features, such as ECG gated single photon emission computed tomography (SPECT), are available as part of the basic system or at extra cost. Following evaluation of tenders received, a recommendation is made to the trust board. This is usually to purchase the cheapest system that meets local specifications, unless there are particular reasons why another system is preferred. Following award of the successful tender, an order is placed for the camera system.

\subsubsection{Camera delivery and installation}

A gamma camera would typically be delivered three months after placement of the order. As a rule, $95 \%$ of the purchase price of the equipment is payable on delivery. Installation times vary according to equipment complexity, but one week would be typical. Immediately after installation, new cameras and computers require careful acceptance testing according to the current Institute of Physics and Engineering in Medicine (IPEM) recommendations. ${ }^{11}$ This is normally completed within 1-2 weeks of system handover, but can take longer. Acceptance testing ensures that the camera meets its stipulated specifications and quoted set of performance parameters, and sets the baseline for subsequent QA procedures. Following satisfactory acceptance testing, the balance of the purchase price is payable (usually $5 \%)$.

The one year warranty period of a camera normally begins only once the first patient has been scanned. Subsequently a service contract with the supplier will be required, at an approximate annual cost of $8-10 \%$ of the purchase price; the exact cost may vary according to the level of service required. The cost of a service contract may be reduced if purchased at the same time as the equipment, but this should not normally be for more than three years in advance (years 2-4).

\subsection{Staffing requirements and training issues}

\subsubsection{Overview}

Many professional groups are involved in nuclear cardiology at a national level, the exact combination varying greatly between departments: nuclear medicine physicians, radiologists, cardiologists, medical physicists, nuclear medicine technologists, radiographers, cardiac physiologists, nurses. Table 4 suggests a minimum staff contingent for running an efficient nuclear cardiology service.

\subsubsection{Medical staff}

Ideally, a nuclear cardiology service should be led by a consultant who combines a sound knowledge of radionuclide imaging with an understanding of clinical cardiology. This could be a nuclear physician or radiologist with a particular insight into cardiology, or a cardiologist with subspecialist training. The Royal College of Radiologists defines four levels of competence for involvement in imaging procedures: 
Table 4 Staff to run a nuclear cardiology service

\begin{tabular}{lll}
\hline Staff type & WTE & Revenue cost \\
\hline Consultant medical staff & 1 & $£ 80 \mathrm{k} /$ year \\
"Technical" (allow for leave, sickness, etc) & 3.5 & $£ 120 \mathrm{k} /$ year \\
Medical physics expert & 0.5 & $£ 20 \mathrm{k} /$ year \\
Admin and clerical & 1 & $£ 20 \mathrm{k} /$ year \\
Total & & $£ 240 \mathrm{k}$ \\
\hline
\end{tabular}

Assuming approximately 2000 MPS studies per year.

The revenue costs shown are estimates given for planning purposes only.

- Level 1-knows about the procedure, and has seen it done

- Level 2-can carry out the procedure under supervision

- Level 3-can carry out the procedure, but cannot report unsupervised

- Level 4-can carry out the procedure and report unsupervised.

Unfortunately, there is no consensus as to what constitutes "level 4" competence for MPS, and the Joint Committee for Higher Medical Training (JCHMT) requirements for "advanced" training for different specialties are very different (table 5). Nuclear medicine and radionuclide radiology specialist registrars require more formal teaching and greater procedure numbers than their cardiology colleagues, though without any formal exposure to clinical cardiology. Cardiology training is about to move away from assessment based on procedure numbers to rely entirely on "competency based" assessment. It is therefore likely that a cardiologist wishing to hold an ARSAC certificate and run his/her own nuclear cardiology service (level 4 competence) would have to demonstrate greater knowledge and experience than is required for a certificate of completion of specialist training (CCST) in cardiology. Often the necessary combination of imaging and cardiological skills can only be achieved in a given hospital through partnership between a cardiologist and a nuclear physician, radiologist, or physicist, at least during reporting sessions.

A recent BCS document on cardiac workforce requirements in the UK estimated that 2.9 whole time equivalent (WTE) "nuclear cardiologists" are required per million population. ${ }^{12}$ For a good sized DGH serving a catchment population of 400000 , this equates to one WTE consultant.

\subsubsection{Technical staff}

There is a nationwide shortage of technical staff within the NHS. Nuclear cardiology services have therefore evolved differently in different hospitals, depending on non-medical staff from a variety of backgrounds: medical physicists, nuclear medicine technologists, radiographers, cardiac physiologists, nurses. ${ }^{13}$ Unfortunately there is currently no unified training scheme or mechanism of accreditation,

Table 5 Current specialist registrar training requirements for "nuclear cardiology" according to specialty

\begin{tabular}{|c|c|c|c|}
\hline Specialty & Training overview & Theory & Practical \\
\hline Cardiology & $\begin{array}{l}4 \text { years basic } \\
\text { cardiology }+2 \text { years } \\
\text { advanced imaging }\end{array}$ & III defined & $\begin{array}{l}75 \text { reports (basic) } \\
+100 \text { hands on } \\
\text { (advanced) }\end{array}$ \\
\hline $\begin{array}{l}\text { Nuclear } \\
\text { medicine }\end{array}$ & 4 years nuclear & Thorough & 500 hands on \\
\hline $\begin{array}{l}\text { Radionuclide } \\
\text { radiology }\end{array}$ & $\begin{array}{l}4 \text { years radiology }+ \\
1 \text { year nuclear } \pm \\
\text { optional } 1 \text { year nuclear }\end{array}$ & Thorough & 500 hands on \\
\hline
\end{tabular}

though the BNCS hopes to address this with a modular voluntary certification scheme in the future.

Image acquisition usually requires a single nuclear medicine technologist, radiographer, or occasionally cardiac physiologist. ${ }^{13}$ The American Society of Nuclear Cardiology (ASNC) has established guidance for technician training in imaging. At least two months should be spent in a dedicated nuclear cardiology department (not easily applicable in the UK), and at least 100 MPS studies should be performed and reconstructed. A certain amount of didactic training is also specified.

Most departments employ two members of staff to perform stress tests, consistent with BCS guidelines. ${ }^{13}{ }^{14}$ Stress remains under the direct supervision of a doctor in the majority of departments, while a radiographer or technician is present to inject the radiopharmaceutical. Many departments have successfully moved towards stress supervised by a specially trained cardiac physiologist, nurse, nuclear medicine technician, or radiographer, usually supported by a colleague, though some kind of remote medical cover remains mandatory. Training for stress testing needs to be tailored to each specific professional group, but there are good precedents for non-doctor supervised stress. Cardiac physiologists have been supervising exercise ECGs for a number of years, with clear guidance available from the BCS. ${ }^{14}$ Many rapid access chest pain clinics are run by nurse practitioners/consultants, who commonly supervise exercise ECGs with a cardiac physiologist. A few nuclear medicine departments currently depend on stress testing supervised by nuclear medicine technologists or cardiac nurse specialists. The training of experienced cardiac physiologists, cardiac nurses, or nuclear medicine technologists to perform pharmacological as well as exercise stress ought to be relatively straightforward, as long as local protocols and training arrangements are in place, perhaps in the form of patient group directives. Trusts must then accept vicarious liability. The recently published national procedure guidelines for MPS may help in the drafting of such protocols. ${ }^{5}$

\subsubsection{Medical physicists}

In a hospital with a large nuclear medicine practice and its own radiopharmacy, it is likely that physics support will be available in house. Otherwise, physics cover may have to be provided (usually at a cost) from a neighbouring centre.

\subsubsection{Administrative and clerical staff}

A busy nuclear cardiology service with a dedicated cardiac camera can perform approximately 2000 MPS studies per year. These patients must have their appointments made and sent, and their telephone queries addressed; they must be greeted on arrival at the department, and their reports typed and sent following the investigation. At least one receptionist/secretary is required to do all this, relieving some of the pressure on the technical staff.

\subsection{Choosing clinical protocols}

\subsubsection{Introduction}

There are several evidence based methods of performing almost every aspect of MPS, reflecting the fact that all methods have both advantages and disadvantages. A complete analysis is beyond the scope of this document, and practical details can be found in the procedure guidelines which have recently been published with the endorsement of the BCS, BNCS, and BNMS. ${ }^{5}$ This section will provide some basic information to inform the decision making process.

\subsubsection{Radiopharmaceuticals and protocols (table 6)}

- Which tracer should be used, thallium-201 $\left({ }^{201} \mathrm{Tl}\right)$ or a technetium-99m $\left({ }^{99 \mathrm{~m}} \mathrm{Tc}\right)$ based agent? 
- If a ${ }^{99 \mathrm{~m}}$ Tc-based agent is to be used, should it be sestamibi or tetrofosmin?

- If a ${ }^{99 \mathrm{~m}}$ Tc-based agent is chosen, should a two day or a one day protocol be used?

- If a one day ${ }^{99 \mathrm{~m}}$ Tc protocol is chosen, should a stress-rest or a rest-stress order be used?

\subsubsection{Approaches to stress (table 7)}

- Who should supervise stress tests? (see 4.4.3)

- What should be the first line form of stress, dynamic exercise or a vasodilator drug?

- When a vasodilator drug is to be used, should this be dipyridamole or adenosine?

\subsubsection{Reliable reporting}

- Who should report the studies? (see 4.4.2)

- How can common errors in reporting be avoided?

- get experience first (regional centre, training CD-ROM)

- always look at the raw data cine (quality, sources of artefact)

- always report from the computer screen, not from a hard copy

- be aware of the common artefacts and do not over-report

- avoid equivocation and try to answer the clinical question

- What should a report look like?

- patient details

- type of study

- indications for study

- procedure

- findings

- conclusion

\section{MAKING THE BUSINESS CASE FOR MPS LOCALLY} 5.1 Introduction

NICE acknowledged professional evidence that national MPS activity should increase from 1200 to 4000 studies per million

Table 6 Advantages of different radiopharmaceutical and protocol options

\begin{tabular}{|c|c|}
\hline $\begin{array}{l}\text { Thallium-201 } \\
\text { Huge literature and familiarity }\end{array}$ & $\begin{array}{l}\text { Technetium- } 99 \mathrm{~m} \text {-based agent } \\
\text { 99mTc available " } 24 / 7 \text { " from } \\
\text { generator }\end{array}$ \\
\hline $\begin{array}{l}\text { Better perfusion tracer } \\
\text { physiologically }\end{array}$ & Lower radiation exposure to patient \\
\hline ? better viability tracer & $\begin{array}{l}\text { Stress imaging can be delayed and } \\
\text { repeated (e.g. for patient movement) }\end{array}$ \\
\hline $\begin{array}{l}\text { Need not be delivered daily } \\
\text { (long half-life) }\end{array}$ & $\begin{array}{l}\text { Better images, particularly in the } \\
\text { obese }\end{array}$ \\
\hline $\begin{array}{l}\text { No preparation required } \\
99 \mathrm{~m} \text { Tc-sestamibi }\end{array}$ & $\begin{array}{l}\text { Gating practical } \\
\text { 99mTc-tetrofosmin }\end{array}$ \\
\hline $\begin{array}{l}\text { Large literature } \\
\text { ? better perfusion tracer } \\
\text { physiologically } \\
\text { Two day } 99 \mathrm{mTc} \text { protocol } \\
\text { Optimal quality images for } \\
\text { stress and rest } \\
\text { Second acquisition unaffected } \\
\text { by first injection } \\
\text { Gating of both acquisitions } \\
\text { possible }\end{array}$ & $\begin{array}{l}\text { No boiling required in preparation } \\
\text { Faster gut clearance for good } \\
\text { imaging } \\
\text { One day }{ }^{99 \mathrm{~m} T c} \text { protocol } \\
\text { More convenient for many patients }\end{array}$ \\
\hline $\begin{array}{l}\text { One day stress-rest order } \\
\text { Rest study avoidable if stress } \\
\text { study normal }\end{array}$ & $\begin{array}{l}\text { One day rest-stress order } \\
\text { No delay required between injections }\end{array}$ \\
\hline
\end{tabular}

Table 7 Advantages of different stress options

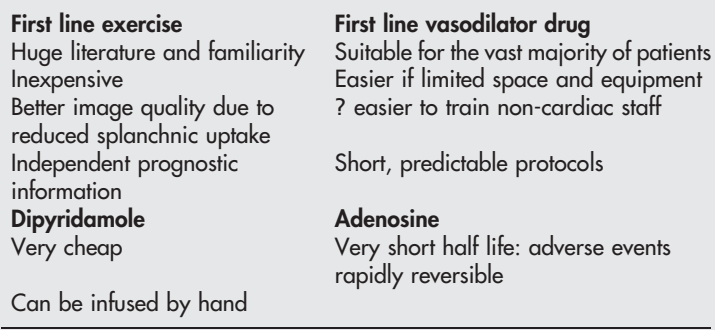
There is no single model for the consideration, prioritisation, and funding of NICE recommendations at local level. Commonly, a

group of PCTs will set up a NICE implementation committee to

population per year based on targets for revascularisation. Suitable waiting times would be six weeks for routine studies and one week for urgent studies, rather than the current national average of 20 weeks. Independently, an NHS improvement plan is defining a new diagnostic capacity strategy, such that by 2008 a "start to treat" target of less than 18 weeks is envisaged. It is possible that in due course some diagnostic intervals will be measured separately. There is no doubt that an ambitious target for all diagnostic tests would have favourable consequences for the expansion of MPS services.

\subsection{Strategic overview to expanding MPS}

The Intercollegiate Standing Committee on Nuclear Medicine has suggested a "hub and spoke" model for service provision. ${ }^{15}$ In the short term, the most effective use of resources may be to ensure that all major nuclear medicine departments have at least two gamma cameras, perhaps with one acting as a dedicated cardiac camera. Such "hub" departments have established protocols, and a concentration of experienced medical and technical staff and physicists. They are the obvious environment in which to train staff from all professional groups, including cardiology specialist registrars. Such a centralised arrangement would be geographically inconvenient for many patients. However, once a given "spoke" district general hospital (DGH) is referring a critical mass of patients, it would become cost effective for it to have its own camera and set up its own local service, probably with support from the "hub". In the medium term there may be a role for mobile nuclear cardiology laboratories, whether run and staffed as an annexe of a "hub" hospital, or used by a "spoke" hospital as a bridge to setting up its own service.

\subsection{Creating a local consensus for expanding MPS}

The development of an MPS service requires the efforts of an energetic lead consultant. Partnerships can be built between cardiologists and nuclear medicine physicians or radiologists, with the aim of integrating MPS into patient care. MPS may rapidly become an indispensable front door service, existing alongside exercise testing in rapid access chest pain clinics, or helping to streamline risk assessment in patients admitted with acute chest pain.

The value of MPS can be established at hospital trust level via quality in practice development teams, the governance committee, and senior management including the medical director. In the community, approaches should be made to local implementation teams and PCTs. There should be patient involvement at all levels. Support may come from other specialties-for example, from a renal transplant network team demanding rapid non-invasive cardiac assessment before transplantation.

\subsection{Process for funding NICE initiatives at PCT level}


consider the issues. The composition of this committee varies from area to area, but a typical membership list might be:

- A lay PCT chair (chair)

- Professional lead (deputy chair)

- Head of health policy of the local public health network

- Members of the professional executive committees (PECs) of the relevant PCTs

- Clinical executive directors of the relevant secondary care trusts

- Special advisors on

- ethics

- law

- finance

- medicine

- pharmaceuticals

- Patient representatives from local patient advice and liaison services (PALS).

PCTs receive no "ring fenced" money to fund NICE recommendations, but are expected to consider them when formulating their local delivery plans. Some PCTs "top slice" funds from their individual budgets which are pooled together and allocated to the NICE implementation committee for prioritisation. Others simply consider the committee's recommendations through the usual commissioning processes.

\subsection{Potential value of MPS for PCTs}

The implementation of NICE guidance for the use of MPS is in line with key areas of the NHS PCT agenda.

- CAD, including angina and myocardial infarction, is an increasing problem-CAD is an increasing health problem, and may be associated with a reduced quality of life and an adverse prognosis. CAD patients are major consumers of health service resources, including hospital admissions.

- The new General Medical Services (nGMS) contract gives incentives for treating diagnosed CAD patients-The nGMS contract came into effect in April 2004. It includes a quality and outcomes framework which will encourage general practices to improve the standard of care they offer. CAD is one of the largest groups of indicators which can bring increased funding. The indicators require that practices hold a register of CAD patients, and that they are appropriately diagnosed and treated.

- CHAI will judge NHS trusts on implementation of NSF and NICE guidance, and according to its own performance indicatorsCHAI is reviewing the implementation of the CAD NSF. It has already assessed acute trusts across both England and Wales, and is currently assessing PCTs: it is due to report in late 2004. CHAI's regular clinical governance reviews assess whether NHS bodies have clear clinical governance plans. Implementation and audit of clinical standards and guidelines, including those set out by NSFs and NICE, are important in this. CHAI's own performance indicators for PCTs and acute and specialist trusts also set standards on $\mathrm{CAD}$ and give incentives for efficient diagnosis.

- The NatPACT PCT Competency Framework also requires good clinical practice in heart disease-The NatPACT PCT Competency Framework provides a tool for PCTs to assess themselves against benchmarks in a wide range of areas. This strongly supports PCTs in acting according to NSFs and NICE guidance in general, and the CHD NSF in particular: "8.7.7 There is an agreed PCT-wide protocol for the systematic assessment, treatment and follow-up of people with CAD".
- An MeReC Bulletin from the National Prescribing Centre (NPC) supports the assessment of cardiovascular risk-The NPC's MeReC bulletin on "Assessing cardiovascular risk" highlights that people with established disease or at high risk of future cardiovascular events need to be identified. GPs can facilitate this by maintaining cardiovascular disease registers and taking a systematic approach to cardiovascular risk assessment.

- Patients should be offered choice over the type of treatment and its delivery-Patient choice has recently become a key NHS priority. In future, patients should be offered choice not only about when and where they receive treatment, but also about what kind of treatment they receive and how it is delivered. This includes rapid and convenient access to diagnostic tests.

\subsection{Constructing a business case for MPS}

- Ensure clinicians have clinical freedom to use MPS where they believe patients will benefit

- Agree implementation of the NICE recommendations for MPS as a local priority, included in the local delivery plan

- Identify any gaps between current practice and the NICE recommendations

- Work with both primary and secondary care to ensure agreement on the most appropriate diagnostic and referral pathway

- Involve patients in decisions about their care.

\subsection{Questions the PCTs will want answered} Clinical

- Where does MPS fit into clinical management?

- What, if anything, will it replace?

- What will it prevent and how do we know?

- What happens if we do not use it?

- How do we treat patients with known or suspected CAD at the moment?

- Are there any guidelines to support a change in practice?

- national level

- local level

- Does MPS work?

- efficacy

- evidence based review

\section{Cost}

- What will the pricing range be?

- Is there supporting health economic data on direct and indirect costs?

- number needed to treat

- quality of life

- impact on other NHS services

- savings over current management

\section{Implementation}

- What will be the number and nature of the patients studied?

- local impact costs

- How will uptake be phased?

- who will be investigated and over what time scale?

- priorities 
- What audit will be arranged to support implementation?

- local action plans on implementation, monitoring, and review

\section{CONCLUSION}

The positive NICE appraisal of MPS has stimulated great interest in the technique. This document is intended as a resource for medical, technical, and managerial staff who are interested in establishing a nuclear cardiology service but are uncertain of the steps involved. It seeks to explain each step to the non-expert, so that he or she can understand the issues and engage in a meaningful dialogue with clinical and medical physics experts, funding bodies, and industry representatives. Together with the template business case for setting up an MPS service which is available electronically (www.bncs.org.uk), it will hopefully prove useful.

Writing group: Constantinos Anagnostopoulos, Royal Brompton Hospital; Glyn Davies, Hull and East Yorkshire Hospitals; Jane Flint, Dudley Group of Hospitals; Mark Harbinson, Queens University Belfast; Andrew Hilson, Royal Free Hospital; Andrew Kelion, Harefield Hospital; Avijiit Lahiri, Wellington Hospital; Eric Lim, Wellington Hospital; Liz Prvulovich, Middlesex Hospital; Nikant Sabharwal, Oxford Deanery; Ann Tweddel, Hull Royal Infirmary; Richard Underwood, Imperial College; Simon Woldman, Ayr Hospital

Advisory group: Roger Boyle, Department of Health; lan Jones, South Derbyshire Acute Hospitals; Kate Latus, Royal Brompton Hospital; Charlie McKenna, Royal Berkshire and Battle Hospitals; Vicky Parkin, Bristol Royal Infirmary; Sarah Powell, Southampton City Primary Care Trust

Correspondence to: Dr Andrew D Kelion, Nuclear Medicine Department, Harefield Hospital, Hill End Road, Harefield, Middlesex UB9 6JH, UK; A.Kelion@rbh.nthames.nhs.uk

\section{REFERENCES}

1 National Institute for Health and Clinical Excellence. http:// www.nice.org.uk/TA073guidance.

- The findings of the NICE technology appraisal of MPS

2 Bax JJ, van der Wall EE, Harbinson MT. Radionuclide techniques for the assessment of myocardial viability and hibernation. Heart 2004;90(suppl V):v26-33

A review of the use of MPS in the assessment of ischaemic left ventricular dysfunction.
3 Underwood SR, Anagnostopoulos C, Cerqueira M et al. Myocardial perfusion scintigraphy: the evidence. Eur J Nucl Med 2004;31:261-91.

- A review of the clinical evidence underpinning the use of MPS; the professional submission to NICE.

4 Anagnostopoulos C, Underwood SR, eds. Myocardial perfusion scintigraphy: the evidence. Heart 2004;90(suppl V):vl-40

- A series of reviews covering many aspects of MPS in clinical practice.

5 Anagnostopoulos $C$, Harbinson M, Kelion AD, et al. Procedure guidelines for radionuclide myocardial perfusion imaging. Heart 2004;90(suppl I):i1-10.

- Procedure guidelines for MPS adopted by BCS, BNCS, and BNMS.

6 De Bono D. Investigation and management of stable angina: revised guidelines 1998. Heart 1999;81:546-58

- BCS guidelines on the investigation and management of stable angina pectoris.

7 Wong $Y$, Rodwell A, Dawkins S, et al. Sex differences in investigation results and treatment in subjects referred for investigation of chest pain. Heart $2001 ; 85: 149-52$.

- Experience of a single chest pain clinic where MPS was not routinely used.

8 Golub RJ, Ahlberg AW, McClellan JR, et al. Interpretive reproducibility of stress Tc-99m sestamibi tomographic myocardial perfusion imaging. I Nucl Cardiol 1999;6:257-69.

- Reproducibility of MPS reporting.

9 Underwood SR, Godman B, Salyani S, et al. Economics of myocardial perfusion imaging in Europe - the EMPIRE study. Eur Heart $J$ 1999:20:157-66.

- The EMPIRE study comparing the cost effectiveness of a range of diagnostic strategies in patients with suspected CAD.

10 Shaw LJ, Hachamovitch R, Berman DS, et al. The economic conseguences of available diagnostic and prognostic strategies for the evaluation of stable angina patients: an observational assessment of the value of precatheterization ischemia. J Am Coll Cardiol 1999;33:661-9.

- A large American study comparing the cost effectiveness of an MPS based diagnostic strategy with direct cardiac catheterisation in patients with suspected CAD.

11 Bolster A, ed. IPEM Report 86. Quality control of gamma camera systems. York: Institute of Physics and Engineering in Medicine, 2003.

- Recommendations for camera QA.

12 Hackett D. Cardiac workforce requirements in the UK. www.bcs.com.

- BCS document suggesting numbers of consultants required in various cardiology subspecialties.

13 Kelion AD, Anagnostopoulos C, Harbinson M, et al. Myocardial perfusion scintigraphy in the UK: insights from the British Nuclear Cardiology Society Survey 2000. Heart 2005:91(suppl IV):iv2-5.

Results of the most recent BNCS survey on MPS in the UK.

14 British Cardiac Society. Protocol for cardiac physiologist managed exercise stress testing. www.bcs.com

- BCS protocol for exercise testing managed by cardiac physiologists without direct medical supervison

15 Intercollegiate Standing Committee on Nuclear Medicine. Nuclear medicine and radionuclide imaging: a strategy for provision in the UK. London: Royal College of Physicians of London, 2003.

- Recommendations for national provision of nuclear medicine services, including MPS. 